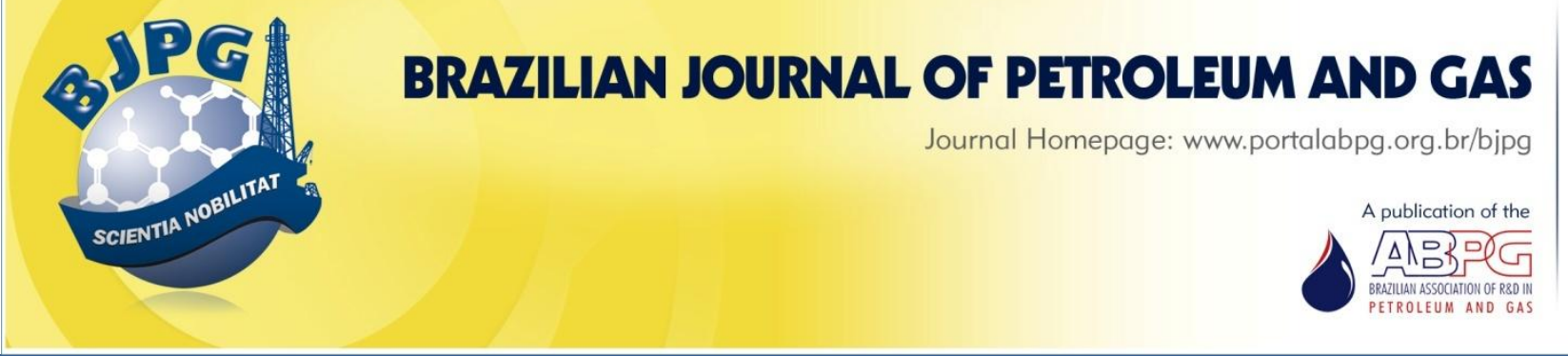

\title{
PARTICLE-SCALE SIMULATION OF THE SEDIMENTATION AND FILTRATION OF DRILLING FLUIDS USING THE DISCRETE ELEMENT METHOD
}

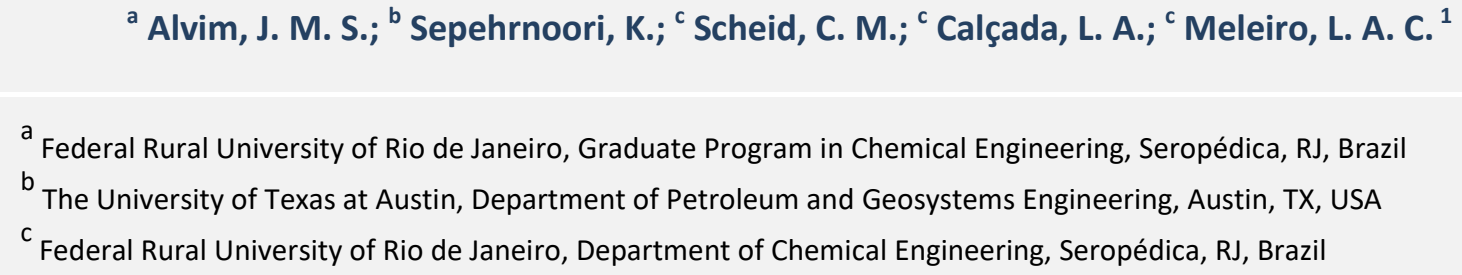

Received: 22.09.2019 / Revised: 11.11.2019 / Accepted: 15.11.2019 / Published on line: 20.12.2019

\begin{abstract}
This paper presents the development of a computational code based on Discrete Element Method (DEM) to simulate the process of solid particles sedimentation and fluid filtration in drilling operations. For both phenomena, the adequate knowledge of the properties and characteristics of the underlying particulate systems represents an aspect of fundamental importance for the safe and efficient design of new drilling fluids and equipment. In this context, this paper presents a particle-scale simulation study, based on DEM, to describe the mechanics of cake formation and growth during the deposition of particulates. Three dimensional (3D) simulations of both processes were carried out to evaluate the proposed algorithm. The cake and the sediment properties, such as thickness, porosity and permeability were quantified and compared, qualitatively and quantitatively, to literature data. The cake properties obtained in this work showed good agreement with data found in similar conditions.
\end{abstract}

\section{KEYWORDS}

cake; permeability; porosity; DEM; particulate

\footnotetext{
${ }^{1}$ To whom all correspondence should be addressed.

Address: Federal Rural University of Rio de Janeiro, Department of Chemical Engineering. Rod. BR 465, km 7, Seropédica - RJ, Brazil.

ZIP Code: 23.897-000 | Phone number: +55 21 3787-3750 | e-mail: meleiro@ufrri.br

doi:10.5419/bjpg2019-0024
} 


\section{INTRODUCTION}

Considering the set of unit operations used for solid-liquid separation, sedimentation and filtration techniques stand out as critical processing steps for a broad spectrum of industry activities, including sectors ranging from chemical and petrochemical segments to fine chemical industries, such as biotechnology and pharmaceutical (Coulson \& Richardson, 1991).

In the case of sedimentation, the basic principle of operation is to use the gravitational field as the driving force to make the suspended solids, typically heavier than the fluid, move toward the bottom of the equipment, promoting the separation and forming a bed of particles known as cake (Perry et al., 1999).

Similarly, the filtration is also an essentially mechanical process, where the separation of the solids present in the liquid occurs through the passage of the suspension by a porous medium or filtering medium. Since filtration is capable of delivering high separation factors, producing clear filtrate and cakes with high solid contents, this operation is commonly used in activities that deal with large volumes of suspension, such as water purification or mining treatment, as well as in industrial applications that use relatively small amounts of suspension, such as the fine chemical industry (Svarovsky, 2000).

For both solid-liquid separation processes, the appropriate knowledge of the physical-chemical properties of the particulate systems, as well as their influence on the growth dynamics and the final structure of the cakes, is essential for the safe and efficient design of new equipment and processes (Ni et al., 2006).

Several methodologies have been developed over the past twenty years to describe and analyze the deposition of particulate solids in liquids, resulting in a vast collection of sedimentation and filtration models available in the literature. One of these models uses particle-scale numerical simulation by means of the DEM (Dong et al., 2006; 2009; Zhu et al., 2007; 2008). In this technique, the particles are described individually, according to a Lagrangian approach, and Newton's equations of motion are used to track the trajectory of each particle over time. The result is high-level information about the microdynamic behavior of the solid phase, which is useful for the better understanding of the sedimentation processes. Such kind of information would not be obtained through the classic phenomenological modeling based on continuum theory or from conventional experimental techniques (Dong et al., 2006; Zhu et al., 2007).

Although DEM has been originally created to deal with particulate systems in the absence of fluids, it has been successfully used by several researchers to reproduce and analyze multiphase processes, extending the discrete approach to solid-gas and solid-liquid flows, as discussed by Crowe et al. (2012), Dong et al. (2006; 2009), Xu and Yu (1997), Xu et al. (2000), and Zhu et al. (2007; 2008).

The main goal of this work is to perform numerical simulations using a DEM-based computational model to simulate the deposition of solids, taking into account the physical-chemical properties of the particulate systems, as well as their influence on growth dynamics and on the final structure of the cakes.

To accomplish this work, three dimensional (3D) simulations of the sedimentation and filtration processes were carried out to evaluate the proposed algorithm and its ability to virtually emulate the behavior of such processes. Cake properties, such as thickness, porosity and permeability were quantified and compared to literature data.

\section{MATERIALS AND METHODS}

\subsection{Mathematical model}

The proposed model based on DEM was used to describe the granular systems by means of a finite set of discrete elements of regular shape, which can be spherical or polygonal. In this approach, granular particles are allowed to interact with each other, with their neighbors (solid walls of an equipment, for example), and/or with the fluid present in a suspension, by changing energy and momentum. Considering such microscopic processes, the solid particles can exhibit two basic movements, translation and rotation, which should be determined by the resulting forces and torques that act on each particle (Zhu et al., 2005; Zhu et al., 2007). 


\subsubsection{Governing equations}

Based on these considerations, the equations for the Newton's second law of motion can be used to describe the dynamic behavior of the particulate material. Considering a particle $i$, belonging to a generic system with $N$ discrete elements, its translation and rotation are described by equations (1) and (2), respectively (Pöschel \& Schwager, 2005).

$m_{i} \frac{d^{2} \boldsymbol{r}_{\boldsymbol{i}}}{d t^{2}}=\boldsymbol{F}_{\boldsymbol{i}}\left(\boldsymbol{r}_{\boldsymbol{j}}, \boldsymbol{v}_{j}, \boldsymbol{\varphi}_{j}, \boldsymbol{\omega}_{\boldsymbol{i}}\right)$

$I_{i} \frac{d^{2} \boldsymbol{\varphi}_{i}}{d t^{2}}=\boldsymbol{T}_{\boldsymbol{i}}\left(\boldsymbol{r}_{\boldsymbol{j}}, \boldsymbol{v}_{j}, \boldsymbol{\varphi}_{\boldsymbol{j}}, \boldsymbol{\omega}_{\boldsymbol{i}}\right) \quad j=1, \ldots, N$

where $\boldsymbol{r}_{i}$ and $\boldsymbol{\varphi}_{i}$ are the vectors of position of the center of mass and angular orientation of the particle $i$, respectively, and $\boldsymbol{F}_{i}$ and $\boldsymbol{T}_{\boldsymbol{i}}$ represent the resultant forces and torques acting on the particle. Mass, $m_{i}$, and the moment of inertia, $l_{i}$, of a perfect spherical particle with density $\rho_{i}$ can be easily obtained through the following relations: $m_{i}=(4 / 3) \pi R_{i}^{3} \rho_{i}$ and $l_{i}=(2 / 5) m_{i} R_{i}^{2}$, respectively (Yang et al., 2000).

If the spatial coordinates and the initial velocities of the particles are known, as well as the appropriate expressions for the evaluation of the resultant forces and torques, equations (1) and (2) can be readily solved by means of an appropriate numerical integration method. As a result, the model provides instantaneous velocities, trajectories, and transient forces acting on the particles step by step in time. This kind of microdynamic information can be directly used in obtaining important parameters for the analysis and quantification of a given physical phenomenon. In the case of filtration, for example, such parameters would be thickness, porosity, and permeability of the cake (Pöschel \& Schwager, 2005).

Different types of interaction forces can be present in the processes of sedimentation and filtration of solid particles, including the gravitational force, the mechanical contact forces, the long-range repulsive and/or cohesive forces (i.e. electrical and van der Waals), and the particlefluid forces. Thus, the resultant force on a given element should be calculated as the vector sum of all contributions, as shown in equation (3) (Zhu et al., 2007).
$\boldsymbol{F}_{\boldsymbol{i}}=\sum_{j=1, j \neq i}^{N} \boldsymbol{F}_{\boldsymbol{i} \boldsymbol{j}}^{\boldsymbol{c}}+\sum_{j=1, j \neq i}^{N} \boldsymbol{F}_{\boldsymbol{i} \boldsymbol{j}}^{\boldsymbol{c}}+\boldsymbol{F}_{\boldsymbol{i}}^{\boldsymbol{f}}+m_{i} \boldsymbol{g}$

where $F_{i j}{ }^{c}$ is the contact force acting on particle $i$ by particle $j ; \boldsymbol{F}_{i j}{ }^{n c}$ is the non-contact force acting on particle $i$ by particle $j ; \boldsymbol{F}_{i}^{f}$ is the particle-fluid interaction force acting on particle $i$, and $\boldsymbol{g}$ is the gravitational acceleration.

Similarly, the resulting torque on particle $i$ can be determined by means of the vector sum of all interaction torques with the other elements of the system with which this particle comes into contact. In this case, the most common contributions include the torque generated by the action of tangential forces and the torque due to rolling friction, according to equation (4) (Yang et al., 2000).

$\boldsymbol{T}_{i}=\sum_{j=1, j \neq i}^{N} \boldsymbol{T}_{i j}^{t}+\sum_{j=1, j \neq i}^{N} \boldsymbol{T}_{i j}^{r}$

where $\boldsymbol{T}_{i j}{ }^{t}$ is the tangential torque acting on particle $i$ by particle $j$ and $\boldsymbol{T}_{i j}{ }^{r}$ is the rolling friction torque acting on particle $i$ by particle $j$.

The models used in this work to calculate the forces and torques mentioned above are summarized in the next topic.

\subsubsection{Modeling of the forces acting on particles}

When two particles of the system collide with each other, their mutual compression or deformation gives rise to the action of mechanical forces in the normal and tangential directions to the contact surface, as shown in Figure 1.

Several models relating these forces with the magnitude of the deformation are available in the literature to represent particle-particle contacts. However, choosing the most appropriate model is still a topic of intense discussions and controversies, since the proper incorporation of the interaction forces is of fundamental importance to the reproduction of the features of a realistic particle bed, as described by Di Renzo and Di Maio (2004), Matuttis and Chen (2014), Pöschel and Schwager (2005), and Zhu et al. (2007). 


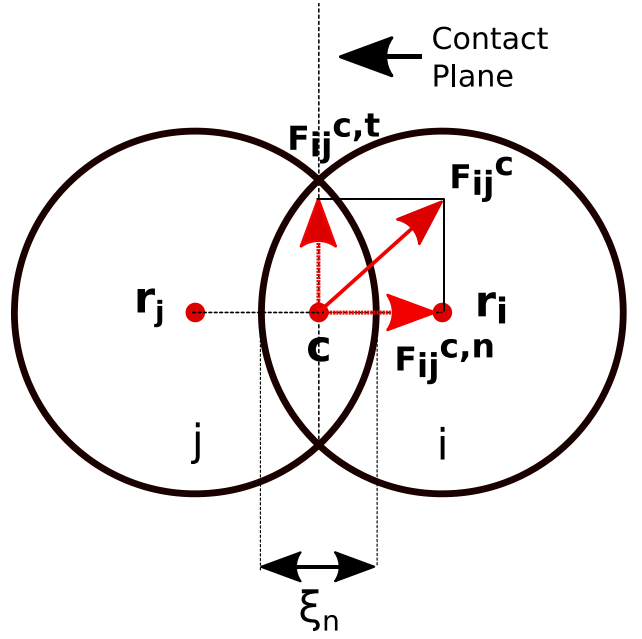

Figure 1. Simplified scheme of the collision between two spherical particles.

Due to its simplicity and ease of implementation, the constitutive model used in this work was the simplified version of the HertzMindlin model for contact between spheres, proposed by Langston et al. (1995). The model employs the Hertzian theory of elasticity to describe the normal contact force component, and a direct force-displacement relationship to handle the tangential component of the force (friction). An additional advantage of this approach is its direct connection to the mechanical properties of the material, such as Young's modulus and Poisson ratio. Equations (5) and (6) are the most common form of this model in the normal and tangential directions, respectively (Zhu et al., 2005; Zhu et al., 2007). The parameters for equations (5) and (6) are summarized in Table 1.

$\boldsymbol{F}_{\boldsymbol{i j}}^{\boldsymbol{c} \boldsymbol{n}}=\frac{4}{3} E \sqrt{R^{e f f}} \xi_{n}^{3 / 2} \boldsymbol{n}_{\boldsymbol{i j}}-\gamma_{n} E \sqrt{R^{e f f} \xi_{n}}\left(\boldsymbol{v}_{\boldsymbol{i} \boldsymbol{j}} \cdot \boldsymbol{n}_{\boldsymbol{i j}}\right) \boldsymbol{n}_{\boldsymbol{i j}}$

$\boldsymbol{F}_{i j}^{c, t}=-\mu\left|\boldsymbol{F}_{i j}^{c, n}\right|\left\{1-\left[1-\frac{\min \left(\left|\xi_{s}\right|, \xi_{s, \max }\right)}{\xi_{s, \max }}\right]^{3 / 2}\right\} \frac{\xi_{s}}{\left|\xi_{s}\right|}$

Since the discrete element method allows the tangential forces to act at the contact point between two particles, instead of their centers of mass, an interaction torque will also be produced whenever a Mindlin-based force model is incorporated into the simulation. This kind of torque can be directly evaluated using equation (7), as the cross product between the radial vector, $\boldsymbol{R}_{i}$, and the tangential force, $\boldsymbol{F}_{i j}^{c, t}$, as described in Zhu et al. (2007).

$T_{i j}^{t}=R_{i} \times F_{i j}^{c, t}$

The rolling friction torque, which includes the effect of asymmetric distributions of normal stress on the particle surfaces, can be adequately modeled according to equation (8), proposed by Zhou et al. (1999).

Table 1. Parameters of the normal and tangential forces models.

\begin{tabular}{cc}
\hline Parameter & Equation \\
\hline Relative velocity at contact & $\xi_{n}=R_{i}+R_{j}-\left|\boldsymbol{r}_{\boldsymbol{i}}-\boldsymbol{r}_{\boldsymbol{j}}\right|$ \\
Elastic modulus & $\boldsymbol{v}_{\boldsymbol{i} \boldsymbol{j}}=\boldsymbol{v}_{\boldsymbol{i}}-\boldsymbol{v}_{\boldsymbol{j}}+\boldsymbol{\omega}_{\boldsymbol{i}} \times \boldsymbol{R}_{\boldsymbol{i}}-\boldsymbol{\omega}_{\boldsymbol{j}} \times \boldsymbol{R}_{\boldsymbol{j}}$ \\
Effective radius & $E=\left(\frac{1-v_{i}^{2}}{Y_{i}}+\frac{1-v_{j}^{2}}{Y_{j}}\right)$ \\
Maximum tangential displacement & $R^{e f f}=\left(R_{i} R_{j}\right) /\left(R_{i}+R_{j}\right)$ \\
Tangential displacement at contact & $\xi_{s, \text { max }}=\mu \xi_{n} \frac{\left(2-v_{i}\right)}{\left(1-v_{i}\right)}$ \\
Relative tangential velocity & $\xi_{\boldsymbol{s}}=\int \boldsymbol{v}_{\boldsymbol{i j}}^{t} d t$ \\
Normal unit vector & $\boldsymbol{v}_{\boldsymbol{i j}}^{t}=\left(\boldsymbol{v}_{\boldsymbol{i j}} \times \boldsymbol{n}_{\boldsymbol{i j}}\right) \times \boldsymbol{n}_{\boldsymbol{i j}}$ \\
& $\boldsymbol{n}_{\boldsymbol{i j}}=\left(\boldsymbol{r}_{\boldsymbol{i}}-\boldsymbol{r}_{\boldsymbol{j}}\right) /\left|\boldsymbol{r}_{\boldsymbol{i}}-\boldsymbol{r}_{\boldsymbol{j}}\right|$
\end{tabular}




$$
\boldsymbol{T}_{\boldsymbol{i} j}^{r}=-\mu_{r} R_{i}\left|\boldsymbol{F}_{\boldsymbol{i j}}^{\boldsymbol{c}, \boldsymbol{n}}\right| \frac{\boldsymbol{\omega}_{\boldsymbol{n}}}{\left|\boldsymbol{\omega}_{\boldsymbol{n}}\right|}
$$

When finer particulates are present in the simulated system, in addition to these interactions of mechanical contact, some long-range forces acquire a significant role in the dynamics of the deposition process (Zhu et al., 2007). Some prominent examples are the van der Waals force, the electrostatic Coulombic force, and the Brownian forces.

Since, in this work, only particles with diameter up to $1 \mu \mathrm{m}$ will be considered, the latter two longrange forces can be reasonably neglected, as discussed on Dong et al. (2006), Yang et al. (2000), and Zhu et al. (2007).

To incorporate the cohesive effect of the van der Waals interaction in the simulations, the discrete element method makes use of the Hamaker theory (1937), which considers, in general terms, that the interactions between individual atoms (or molecules) are additive, so that the resulting van der Waals interactions between macroscopic bodies can be obtained by integrating over all pairs of atoms (Hamaker, 1937; Zhu et al., 2007).

The cohesive force model used in this work is the same described by Israelachvili (1991) and Yang et al. (2000) for smooth spheres, as shown in equation (9).

$F_{i j}^{v}=$

$=-\frac{A}{6} \frac{64 R_{i}^{3} R_{j}^{3}\left(h+R_{i}+R_{j}\right)}{\left(h^{2}+2 R_{i} h+2 R_{j} h\right)^{2}\left(h^{2}+2 R_{i} h+2 R_{j} h+4 R_{i} R_{j}\right)} \boldsymbol{n}_{i j}$

The parameter $A$, known as Hamaker constant, is not easy to quantify since it depends on several factors related to the physical and chemical properties of the particles. In numerical modeling using the DEM, this property is often treated as a lumped parameter to be determined empirically (Dong et al., 2006; Yang et al., 2000).

As can be observed from equation (9), as the distance of separation between two particles, $h$, tends to zero, the cohesive force tends to infinity, introducing a problem of singularity in the model. In this work, the problem was solved by specifying a distance threshold, $h_{\min }$, to avoid the occurrence of an excessive attractive force without physical meaning in simulations. According to the literature, typical values for this parameter range from 0.165$1 \mathrm{~nm}$ (Israelachvili, 1991; Yang et al., 2000; Zhu et al., 2007).

In separation processes, particulate solids also interact with the fluid, giving rise to numerous particle-fluid forces, especially when the fluid is a liquid instead of a gas (Zhu et al., 2007). Thus, in order to obtain more realistic and accurate results, it is necessary that such forces are adequately incorporated into simulations. In this work, three particle-fluid force models adopted in previous works of Dong et al. (2006) were also used: buoyancy, lift, and drag force models (equations 10 through 12).

$$
\begin{aligned}
& \boldsymbol{F}_{\boldsymbol{i}}^{\boldsymbol{b}}=m_{i} \boldsymbol{g}\left(\frac{\rho_{l}}{\rho_{s}}\right) \\
& \boldsymbol{F}_{\boldsymbol{i}}^{\boldsymbol{l}}=\frac{\pi}{8} \rho_{l} R_{i}^{3}\left(1-\phi_{i}\right)\left[\left(0.5 \boldsymbol{\nabla} \times \boldsymbol{u}_{\boldsymbol{l}}-\boldsymbol{\omega}_{\boldsymbol{i}}\right) \times\left(\boldsymbol{u}_{\boldsymbol{l}}-\boldsymbol{v}_{\boldsymbol{i}}\right)\right] \\
& \boldsymbol{F}_{\boldsymbol{i}}^{\boldsymbol{d}}=\boldsymbol{f}_{\boldsymbol{f} \mathbf{0}, \boldsymbol{i}} \phi_{i}^{-(\chi+1)}
\end{aligned}
$$

where the parameters of the drag force model are compiled in Table 2.

Table 2. Parameters of the drag force model.

\begin{tabular}{cc}
\hline Parameter & Equation \\
\hline Drag force on an isolated particle & $\boldsymbol{f}_{\boldsymbol{f} \mathbf{0 , \boldsymbol { i }}}=0.5 c_{d 0, i} \rho_{l} \pi R_{i}^{2} \phi_{i}^{2}\left|\boldsymbol{u}_{\boldsymbol{l}}-\boldsymbol{v}_{\boldsymbol{i}}\right|\left(\boldsymbol{u}_{\boldsymbol{l}}-\boldsymbol{v}_{\boldsymbol{i}}\right)$ \\
Empirical coefficient & $\chi=3.7-0.65 \exp \left[-\frac{\left(1.5-\log _{10} \operatorname{Re}_{i}\right)^{2}}{2}\right]$ \\
Particle-fluid drag coefficient & $c_{d 0, i}=\left(0.63+\frac{4.8}{R e_{i}^{0.5}}\right)^{2}$ \\
Particle Reynolds number & $R e_{i}=\frac{2 \rho_{l} R_{i} \phi_{i}\left|\boldsymbol{u}_{\boldsymbol{l}}-\boldsymbol{v}_{\boldsymbol{i}}\right|}{\mu_{l}}$ \\
\hline
\end{tabular}


In particulate systems, unlike the case of an isolated particle, the presence of a great number of particles decreases the available space for the fluid, giving rise to a sharpened velocity profile and to a higher shear stress on the surface of the particle. The proposed drag force model incorporates this effect through the local porosity, $\phi_{i}$, and the coefficient, $\eta$, associated with both the properties of the liquid and the flow conditions (Crowe et al., 2012; Dong et al., 2006).

The chosen models are well reported and have already been widely used in the literature to describe the dynamics of sedimentation and filtration processes, as discussed in Dong et al. (2006; 2009) and Zhu et al. (2007).

\subsubsection{Contact detection}

Once defined the appropriate force models, the calculation of the sum of forces and torques acting on each particle should be performed, in accordance to equations ( 3 ) and (4). This step is the central point of the DEM, since the computational efficiency of any simulation is basically determined by the efficiency with which the contacts are detected and quantified (Matuttis \& Chen, 2014; Munjiza \& Andrews, 1998).

The simplest way to accomplish this task is through a binary-check algorithm where, at each time step, the possibility of collision between all pairs of particles is checked. However, for shortrange interparticle interactions, such as collision forces, most of these checks are unnecessary, since the simulated particles often are too far apart from each other (Munjiza \& Andrews, 1998; Pöschel \& Schwager, 2005).

Due to this problem, several algorithms available in the literature are used to improve the contact detection procedure (Allen \& Tildesley, 1987; Munjiza \& Andrews, 1998; Pöschel \& Schwager, 2005).

In this work the chosen methodology was the No Binary Search (NBS) contact detection algorithm proposed by Munjiza and Andrews (1998), which reduces the computational cost of the simulations by mapping the simulated discrete elements through a mesh of individual cells, and restricting the contact check only to the pairs of particles which are close neighbors, as illustrated in Figure 2.

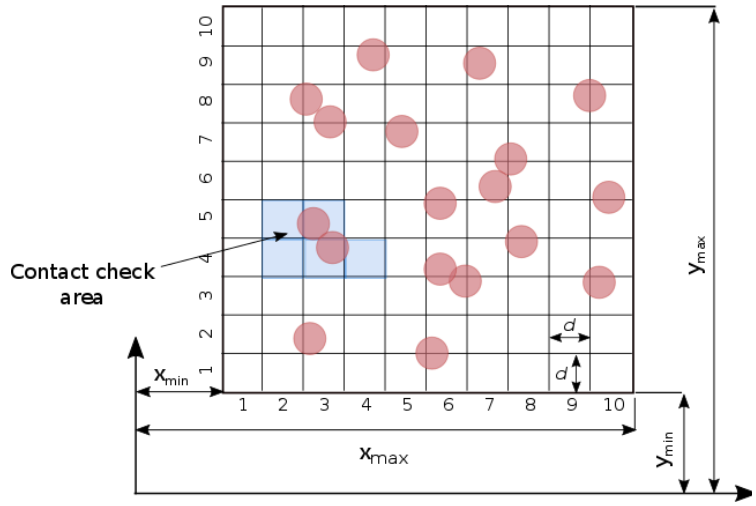

Figure 2. NBS contact detection algorithm for a 2D particulate system. The necessary range of the search for particles in neighboring cells is indicated in blue.

Since this algorithm can be highly demanding on memory used (RAM), it is more suitable for simulating systems with large amounts of particles. However, the NBS algorithm demands a CPU time proportional to the total number of discrete elements and is relatively easy to program (Munjiza \& Andrews, 1998).

\subsubsection{Numerical integration method}

As pointed out in section 2.1, to solve the system of differential equations of motion, it is necessary to use an appropriate numerical integration method. Considering the dynamics of the granular systems, explicit integration methods are often used, since they do not require excessive evaluations of the interaction forces at each time step. The goal is to make the numerical code more efficient in terms of computational cost, since the contact detection step is the biggest performance bottleneck of the discrete element method (Matuttis \& Chen, 2014; Pöschel \& Schwager, 2005).

Due to its robustness and tradeoff between good accuracy and low computational cost in problems involving discrete elements, the numerical integration approach used in this work was the fifth-order Gear's prediction-correction algorithm (Allen \& Tildesley, 1987; Matuttis \& Chen, 2014; Pöschel \& Schwager, 2005).

The particles interactions with the boundaries were modeled by building up rigid and stationary walls, with the same set of physical properties of 


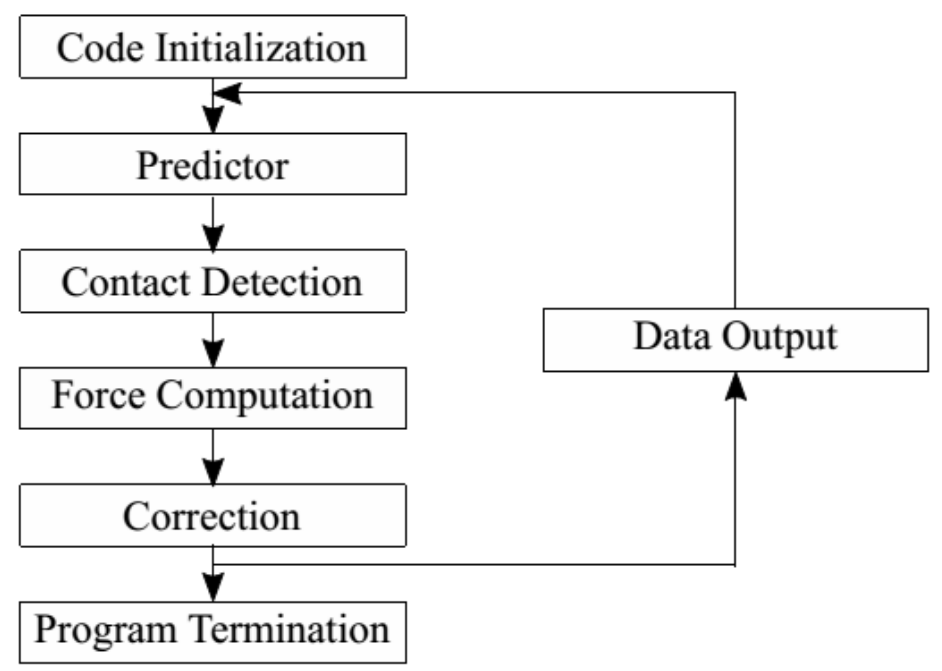

Figure 3. Basic flowchart of the DEM algorithm used in this work.

the simulated particles. There are other ways to specify boundary conditions in DEM modeling that can also be found in the literature, with especial reference to the so-called "periodic conditions," often used to avoid boundary effects on the results of the simulation (Allen \& Tildesley, 1987; Pöschel \& Schwager, 2005; Yang et al., 2000).

In order to start the numeric solution by using Gear's method, a set of initial conditions must be specified, i.e., the spacial coordinates and angular orientations of the particles, as well as their respective temporal derivatives of higher order, $\boldsymbol{r}_{i}^{(n)}$ and $\boldsymbol{\varphi}_{i}^{(n)}$, for $\boldsymbol{n}=0,1,2, \ldots$, at time $t_{0}$ (Pöschel \& Schwager, 2005). In this work, the initial conditions were randomly generated, as described in section 3.

\subsection{Code description}

Figure 3 shows the flowchart of the DEM algorithm implemented in this work, where the computational code was used to reproduce experimental results related in literature, as well as to simulate and analyze the dynamic processes of cake formation and growth in sedimentation and filtration operations.

The first step is to define the geometry of the system, including the coordinates of the particles and their time derivatives, as well as the boundary conditions that limit the simulated area. In addition, the values of the properties of the solid and the fluid, which will be used for the evaluation of the force models presented previously, should also be provided. As a result, the program runs dynamically, reproducing the loop of integration represented in Figure 3 for a predefined number of time steps.

At the beginning of each cycle, the spatial coordinates and time derivatives of the particles are calculated at time $t+\Delta t$ by using the Taylor series expansion of the actual values at time $t$, corresponding to the so-called prediction step of the Gear method. Based on these values, the contact detection algorithm is called to identify the pairs of colliding particles, and a list of these particles is stored.

Once the resultant forces and torques acting on the particles are computed based on the contact list, the second stage of the Gear's method (correction) is called to correct the spatial coordinates and time derivatives obtained in the prediction stage. The data stored during the simulation loops are used to obtain useful information for process engineering analysis, such as cake thickness, porosity, and permeability.

The execution of the program continues following the loop, i.e., updating positions and velocities of the particles, until a previously established final time is reached.

\subsubsection{Simulation parameters}

The particulate system used to represent the sedimentation and filtration processes was modeled through a finite set of discrete spherical particles. Their positions and velocities were randomly generated, without initial mechanical 
Table 3. Set of simulation parameters and physical properties (Dong et al., 2006).

\begin{tabular}{lll}
\hline Parameter & Value & Unity \\
\hline Number of particles, $N$ & 1000 & - \\
Particle diameter, $d_{p}$ & $150-500$ & $\mu \mathrm{m}$ \\
Solid density, $\rho_{s}$ & 2460 & $\mathrm{~kg} / \mathrm{m}^{3}$ \\
Liquid density, $\rho_{l}$ & 1000 & $\mathrm{~kg} / \mathrm{m}^{3}$ \\
Liquid viscosity, $\mu_{l}$ & 1 & $\mathrm{cP}$ \\
Young's modulus, $Y$ & $1.0 \times 10^{6}$ & $\mathrm{~N} / \mathrm{m}^{2}$ \\
Poisson ratio, $v$ & 0.29 & - \\
Normal damping coefficient, $\gamma_{n}$ & $2.0 \times 10^{-5}$ & - \\
Sliding friction coefficient, $\mu$ & 0.3 & - \\
Rolling friction coefficient, $\mu_{r}$ & 0.002 & - \\
Hamaker constant, $A$ & $6.5 \times 10^{-20}$ & $\mathrm{~J}$ \\
Minimum separation, $h_{\min }$ & 1 & $\mathrm{~nm}$ \\
Initial filtrate flow rate, $q_{0}$ & 0.01 & $\mathrm{~m} / \mathrm{s}$ \\
Average cross-flow velocity, $<u_{\mid x}>$ & 0.001 & $\mathrm{~m} / \mathrm{s}$ \\
Pressure drop, $\Delta \mathrm{P}$ & 2 & $\mathrm{kPa}$ \\
\hline
\end{tabular}

contact, within a rectangular shaped control volume with dimensions $2.5 \mathrm{~cm} \times 2.5 \mathrm{~cm} \times 10 \mathrm{~cm}$, for the sedimentation and static filtration cases, and with dimensions $10 \mathrm{~cm} \times 2.5 \mathrm{~cm} \times 2.5 \mathrm{~cm}$ for the cross-flow filtration case. During the simulation process, the particles interacted with each other and with the surrounding fluid according to the governing laws described in equations (5) through (12), exchanging energy and momentum. The particles were allowed to settle down under the action of gravity and particle-fluid forces until they reached a mechanically stable position, that is, with average velocity close to zero. The basic parameters and physical properties used in the simulations (Table 3 ) were specified considering a system composed of a set of glass beads immersed in water and were based on the work by Dong et al. (2006).

To calculate the drag and lift forces, local porosity around the solid particle, $\phi_{i}$, was obtained by dividing the control volume in horizontal cells, each of them with a height equivalent to $0.25 \mathrm{~cm}$. The porosities of these slices were calculated on the basis of the volume of the particles inside each of them. The magnitudes of the time steps used in the simulations, determined by trial and error procedure, varied from $1.0 \times 10^{-5} \mathrm{~s}$ and $1.0 \times 10^{-6} \mathrm{~s}$.

\section{RESULTS AND DISCUSSIONS}

The results for the simulations of sedimentation and filtration processes of a spherical particles system (physical properties are listed in Table 3) are presented in sequence.

In both processes, the cake thickness (height attained by the particle bed) was dynamically calculated as the difference between the higher and lower heights where horizontal planes can intercept mechanically stable particles. In addition, the cake porosity, a parameter commonly used to describe the structure of porous beds, was also quantified as a function of time during the deposition process. In order to avoid top and bottom effects on the results, the calculation of this property was performed based on a central slice of the cake, instead of its total volume, as was suggested by Abreu et al. (2003). The equations (13) and (14) were used to calculate the cake porosity.

$\phi_{c}=1-\frac{\sum_{i=1}^{N} f\left(z_{\min }, Z, z_{\max }\right) V_{i}}{A_{s}\left(z_{\max }-z_{\min }\right)}$

$f\left(z_{\text {min }}, z, z_{\text {max }}\right)=\left\{\begin{array}{ccc}1 & \text { if } & z_{\text {min }} \leq z \leq z_{\text {max }} \\ 0 & \text { otherwise }\end{array}\right.$ 

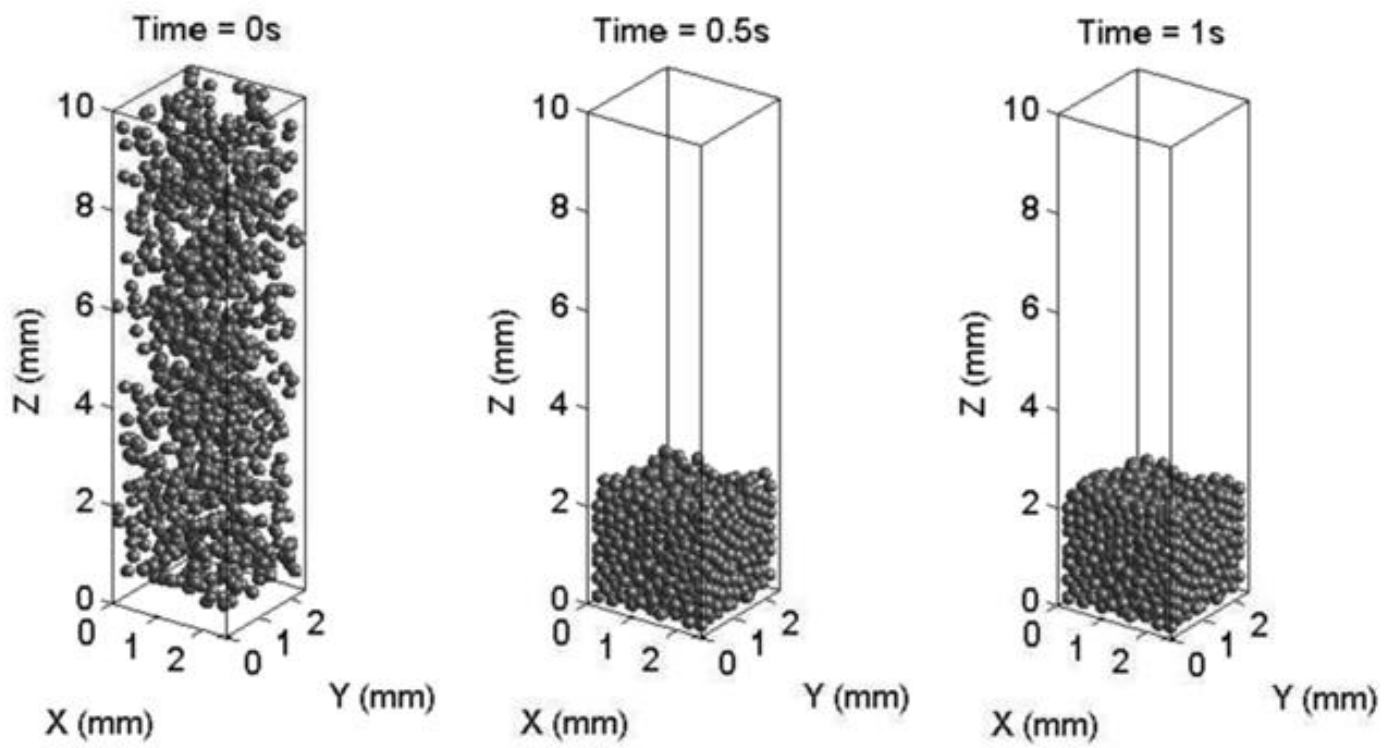

Figure 4. Simulation of the dynamics $(0 \mathrm{~s}, 0.5 \mathrm{~s}$, and $1 \mathrm{~s}$, from left to right) of the sedimentation process with 1000 spherical particles $\left(d_{p}=250 \mu \mathrm{m}\right)$.

where $z_{\max }$ and $z_{\min }$ represent the height limits of the control volume used for determination of the cake porosity, $A_{s}$ represents the cross-sectional area and $V i$ the volume occupied by the particle $i$.

\subsection{Simulation of the sedimentation processes}

In this case study, the liquid velocity was set to zero, since the movement of the liquid phase is very slow if compared to the motion of the settling particles. On the other hand, the displacement of the solid particles was considered to be threedimensional. Figure 4 exhibits the results of the simulation of the temporal evolution of a 3D sedimentation process for 1000 monodispersed spherical particles with diameter $250 \mu \mathrm{m}$.

It can be seen from Figure 4 that after $1 \mathrm{~s}$ every particle has already settled, reaching a mechanically stable position. This conclusion is corroborated through the temporal profiles of the cake thickness and porosity, presented in Figures 5 and 6 , respectively.

As can be seen from Figure 5, the cake thickness presents a linear growth of approximately between Os and $0.4 \mathrm{~s}$, which is qualitatively in good agreement with the current knowledge about sedimentations, as well as to the numerical results obtained by Dong et al. (2003; 2009).

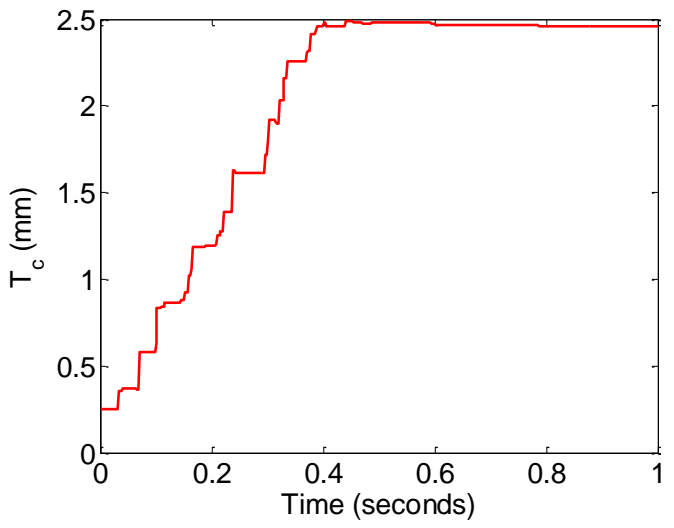

Figure 5. Evolution of the cake thickness with time during the sedimentation process.

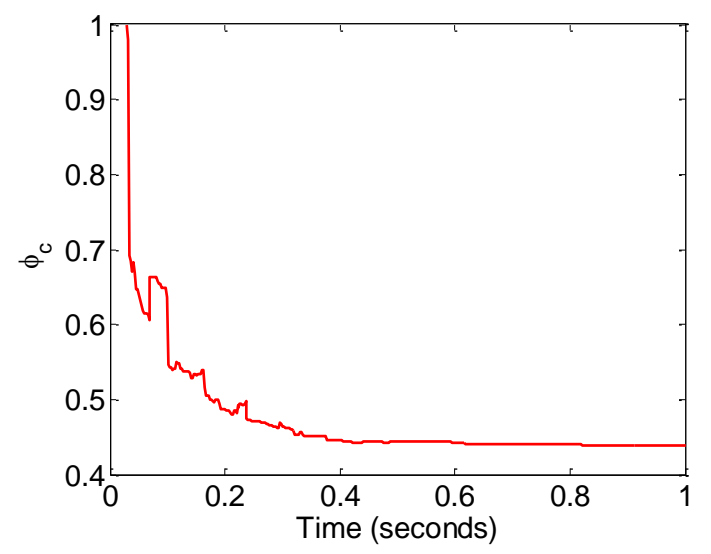

Figure 6. Evolution of the cake porosity with time during the sedimentation process. 


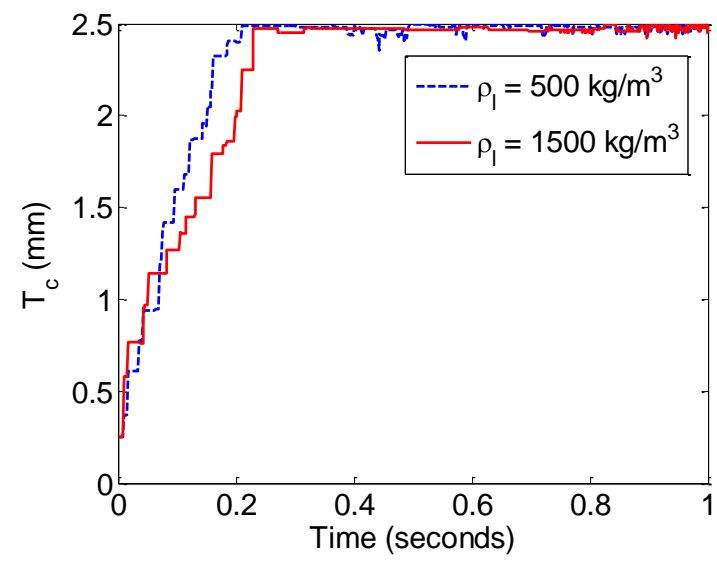

Figure 7. Dynamic behavior of the cake thickness for different liquid densities when $d_{p}=250 \mu \mathrm{m}, A=$ $0.57 \times 10^{-20} \mathrm{~J}$ and $\mu_{l}=1.238 \mathrm{cP}$, according to the simulation conditions presented in Dong et al.

(2003; 2006; 2009).

The cake porosity (Figure 6) steeply decreases at the beginning of the process and reaches a final value around $43 \%$, which also is in agreement with the results previously reported in similar conditions of simulation by Dong et al. $(2003 ; 2006 ; 2009)$ and Jin and Patzek (2003).

A validation of the algorithm described in this work was carried out by simulations of sedimentation processes with different liquid density, viscosity, and Hamaker constant, in similar conditions to those related by Dong et al. (2003; 2006; 2009). These authors reproduced the deposition process of glass beads in a liquid mixture by means of physical and computational

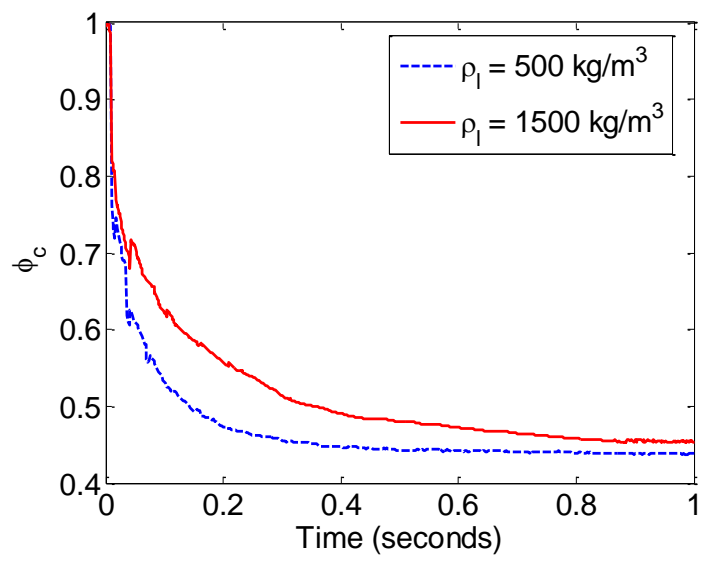

Figure 8. Dynamic behavior of the cake porosity for different liquid densities when $d_{p}=250 \mu \mathrm{m}, A=$ $0.57 \times 10^{-20} \mathrm{~J}$ and $\mu_{l}=1.238 \mathrm{cP}$, according to the simulation conditions presented in Dong et al.

(2003; 2006; 2009).

experiments, obtaining good agreement between the results. Figures 7 and 8 show cake thickness and porosity as a function of time under two different liquid densities, $500 \mathrm{~kg} / \mathrm{m}^{3}$ and 1500 $\mathrm{kg} / \mathrm{m}^{3}$, according to the simulation conditions proposed in Dong et al. $(2003 ; 2006 ; 2009)$ for the sedimentation process of 2250 monodispersed spherical particles with $d_{p}=250 \mu \mathrm{m}$.

The results presented in the figures above show that the increase of liquid density tends to increase both sedimentation time and cake porosity. This effect is directly associated to the presence of enhanced upward fluid-particle forces acting in a denser fluid, which are capable of counteracting

Table 4. Results for the packing fraction provided by this work and by Dong et al. (2006).

\begin{tabular}{ccccc}
\hline Fluid & $\rho_{l}\left(\mathrm{~kg} / \mathrm{m}^{3}\right)$ & $\begin{array}{c}\phi_{s} \\
\text { (This work) }\end{array}$ & $\begin{array}{c}\phi_{s} \\
\text { (Dong et al., 2006) }\end{array}$ & Error (\%) \\
\hline 1 & 2403.1 & 0.4875 & 0.5487 & -11.15 \\
2 & 2352.0 & 0.5223 & 0.5682 & -8.07 \\
3 & 2295.9 & 0.5375 & 0.5772 & -6.87 \\
4 & 2204.1 & 0.5554 & 0.5861 & -5.24 \\
5 & 2102.0 & 0.5593 & 0.5921 & -5.54 \\
6 & 1994.9 & 0.5643 & 0.5936 & -4.94 \\
7 & 1801.0 & 0.5866 & 0.5966 & -1.68 \\
8 & 1602.0 & 0.5835 & 0.5981 & -2.45 \\
9 & 1398.0 & 0.5825 & 0.6011 & -3.10 \\
10 & 1204.1 & 0.5861 & 0.6041 & -2.98 \\
11 & 1005.1 & 0.5896 & 0.6056 & -2.65 \\
12 & 795.9 & 0.5991 & 0.6056 & -1.08 \\
13 & 500.0 & 0.5977 & 0.6071 & -1.55 \\
\hline
\end{tabular}




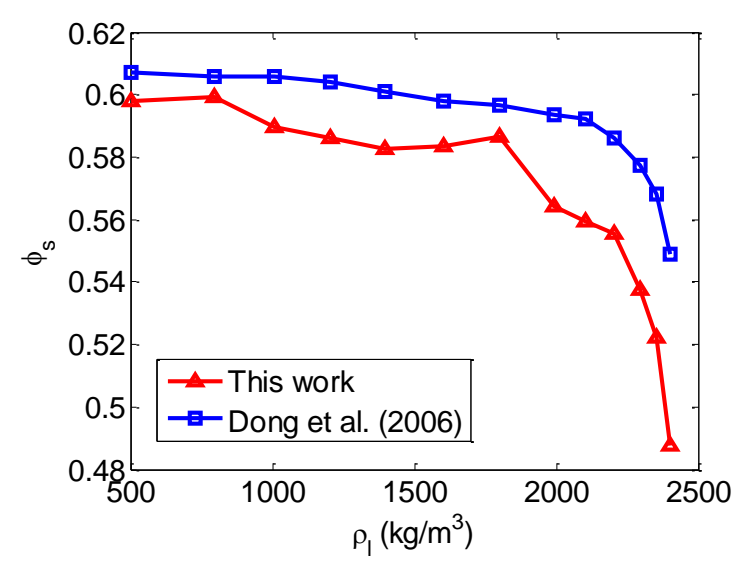

Figure 9. Comparison between steady state results for the cake packing fraction provided by this work and by Dong et al. (2006).

the gravity force more efficiently, thus, enabling the particles to quickly reach the terminal velocity as described in Dong et al. (2003; 2009).

Table 4 shows the results for the packing fraction provided by this work and by Dong et al. (2006) for several fluids with different densities. Percent deviations were evaluated taking as reference the data from Dong et al. (2006).

The results obtained in this work, shown in Table 4, agree reasonably well with data reported by Dong et al. (2006), since deviation remains below $10 \%$ in most of the fluids, except for fluid density above approximately $2352.0 \mathrm{~kg} / \mathrm{m}^{3}$.

A graphical visualization of these results is displayed in Figure 9, where it is possible to notice that both models exhibit the same tendency, since as the liquid density increases the packing fraction decreases. This tendency becomes more evident in simulations where the difference between the liquid and solid densities is small. Besides, this behavior clearly agrees with the theoretical knowledge on sedimentation processes, since the increase of the liquid density changes the force balance that governs the particles movement in order to neutralize the gravitational force, favoring the formation of cakes with higher porosity (Dong et al., 2006; Dong et al., 2009; Svarovsky, 2000).

It is also possible to notice that the model proposed in this work tends to underestimate all the packing fraction values, predicting the formation of less compact cakes in comparison with the results from Dong et al. (2006). Some issues involved with the implementation of the proposed code could be associated with this behavior, but it is possible to say that two of the most important are the differences between the systems sizes and types of boundary conditions used. Dong et al. (2006) simulated a system with approximately three times the number of particles used here. They applied periodical boundary conditions along the two horizontal directions to avoid lateral wall effects, thus, enabling the formation of a more homogeneous packing.

Figures 10 to 13 also show cake thickness and porosity as a function of time for two different

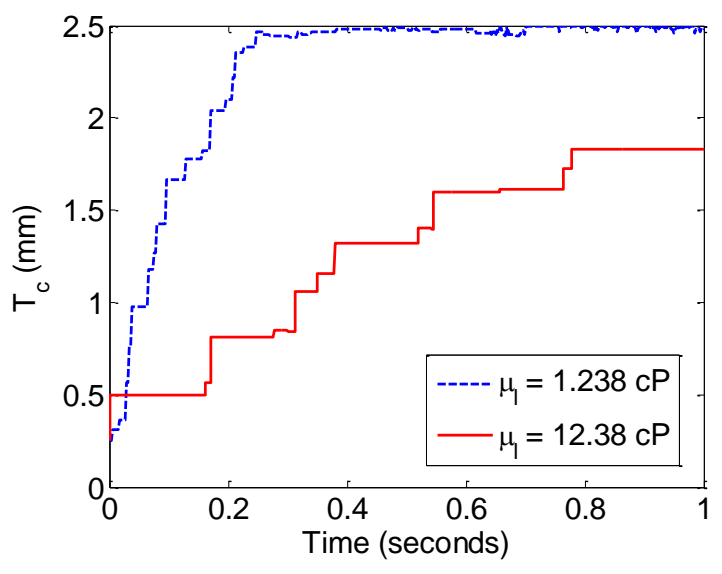

Figure 10. Dynamic behavior of the cake thickness for different liquid viscosities when $d_{p}=250 \mu \mathrm{m}, A=$ $0.57 \times 10^{-20} \mathrm{~J}$ and $\rho_{l}=1000 \mathrm{~kg} / \mathrm{m}^{3}$, according to the simulation conditions presented in Dong et al.

(2003; 2009).

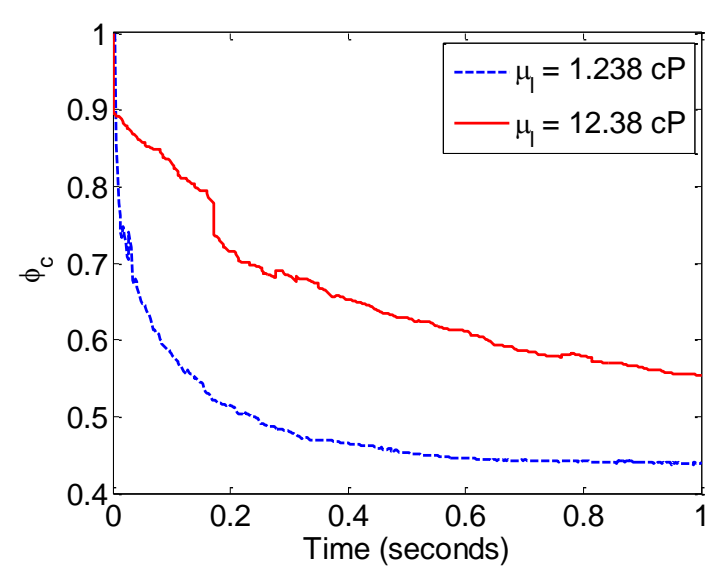

Figure 11. Dynamic behavior of the cake porosity for different liquid viscosities when $d_{p}=250 \mu \mathrm{m}, A=$ $0.57 \times 10^{-20} \mathrm{~J}$ and $\rho_{l}=1000 \mathrm{~kg} / \mathrm{m}^{3}$, according to the simulation conditions presented in Dong et al.

(2003; 2009). 


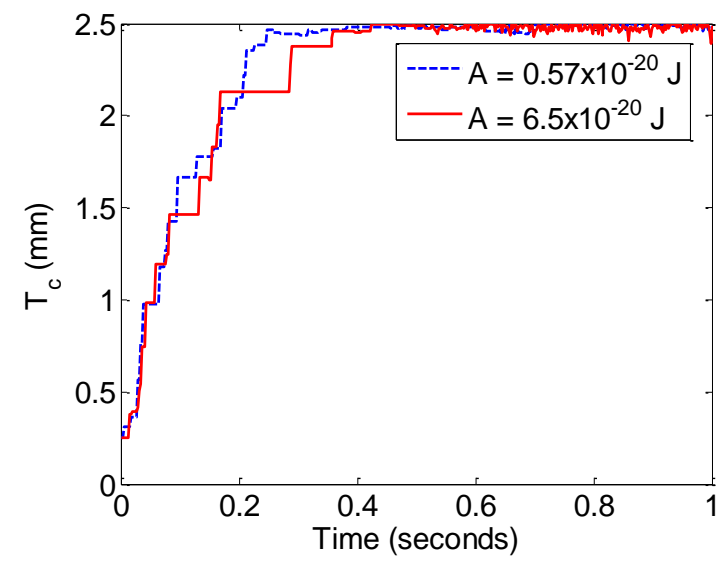

Figure 12. Dynamic behavior of the cake thickness for different Hamaker constants when $d_{p}=250 \mu \mathrm{m}$, $\mu_{l}=1.238 \mathrm{cP}$ and $\rho_{l}=1000 \mathrm{~kg} / \mathrm{m}^{3}$, according to the simulation conditions presented in Dong et al.

(2003; 2009).

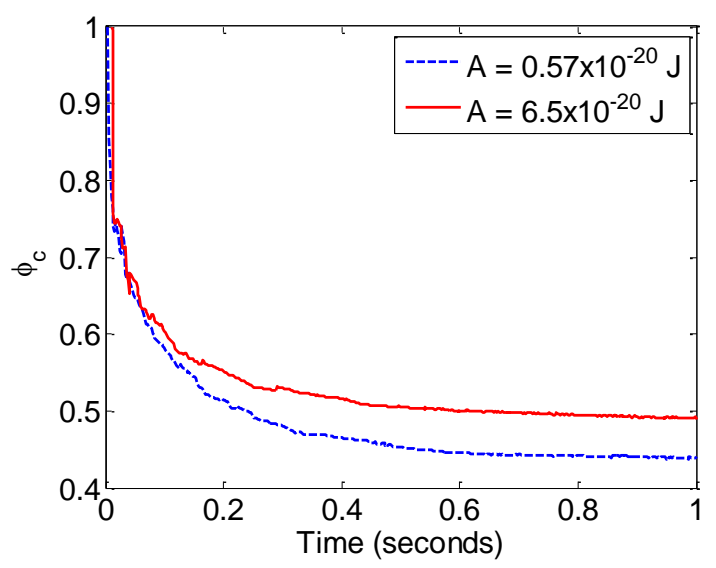

Figure 13. Dynamic behavior of the cake porosity for different Hamaker constants when $d_{p}=250 \mu \mathrm{m}, \mu_{l}=$ $1.238 \mathrm{cP}$ and $\rho_{l}=1000 \mathrm{~kg} / \mathrm{m}^{3}$, according to the simulation conditions presented in Dong et al.

(2003; 2009).

liquid viscosities, $1.238 \mathrm{cP}$ and $12.28 \mathrm{cP}$, and two different Hamaker constants, $0.57 \times 10^{-20} \mathrm{~J}$ and $6.5 \times 10^{-20} \mathrm{~J}$, according to the simulation conditions proposed in Dong et al. $(2003 ; 2009)$ for the sedimentation process of 2250 monodispersed spherical particles.

From the results above one can observe that increasing liquid viscosity also increases sedimentation time and cake porosity. Again, this behavior is associated directly to stronger fluidparticle forces (especially the drag force) that decelerate the particles during the settling process. The Hamaker constant seems to have little effect on the sedimentation time. Nevertheless, by limiting the particles rearrangement once they reach the cake surface, this parameter tends to cause larger differences in cake porosity (Figure 13). Both conclusions are qualitatively in good agreement with the results by Dong et al. (2003; 2009).

\subsection{Simulation of the filtration processes}

The code developed in this work was also used to simulate the static filtration process, operating at constant pressure drop and using the same set of physical conditions described in the sedimentation case (see Table 3). However, now the liquid flow is assumed to be one dimensional along the filtration direction (-z direction) and the liquid velocity relates to the filtrate flow rate according to $\boldsymbol{u}_{\boldsymbol{l}}=(0,0,-q)$.

Instead of monodispersed particles, the size distribution given by equations (15) and (16) was specified, where $Z$ is a random number that belongs to the range [0,1] (Zhu et al., 2005).

$$
\begin{aligned}
& p(R)=\frac{R_{\text {min }} R_{\text {max }}}{R_{\text {max }}-R_{\text {min }}}\left(\frac{1}{R^{2}}\right) \\
& R=\frac{R_{\text {min }} R_{\text {max }}}{R_{\text {max }}-Z\left(R_{\max }-R_{\text {min }}\right)}
\end{aligned}
$$

According to this probability distribution, the radii of the particles are chosen from the interval $\left(R_{\min }, R_{\max }\right)$ in such a way that the total mass of all particles from a certain size interval is the same for all sizes, thus, ensuring that neither large nor small particles dominate the system Zhu et al. (2005).

The surface of the filtering medium was modeled as a layer of fixed solid particles, with the same mechanical properties of the suspended ones. Figure 14 presents the different time instants $(0 \mathrm{~s}, 0.5 \mathrm{~s}$, and $1 \mathrm{~s})$ of the simulated filtration process operating at a constant pressure drop of 2 $\mathrm{kPa}$. The initial filtrate flow rate, $q_{0}$, was considered to be $1 \mathrm{~mm} / \mathrm{s}$.

Solid particles with size distribution in the range of 150-250 $\mu \mathrm{m}$ were introduced gradually in the simulated region at a constant rate of 0.00125 particles/time step in an attempt to virtually reproduce the continuous operation mode.

According to these diagrams, a large number of particles has already settled on the surface of the 

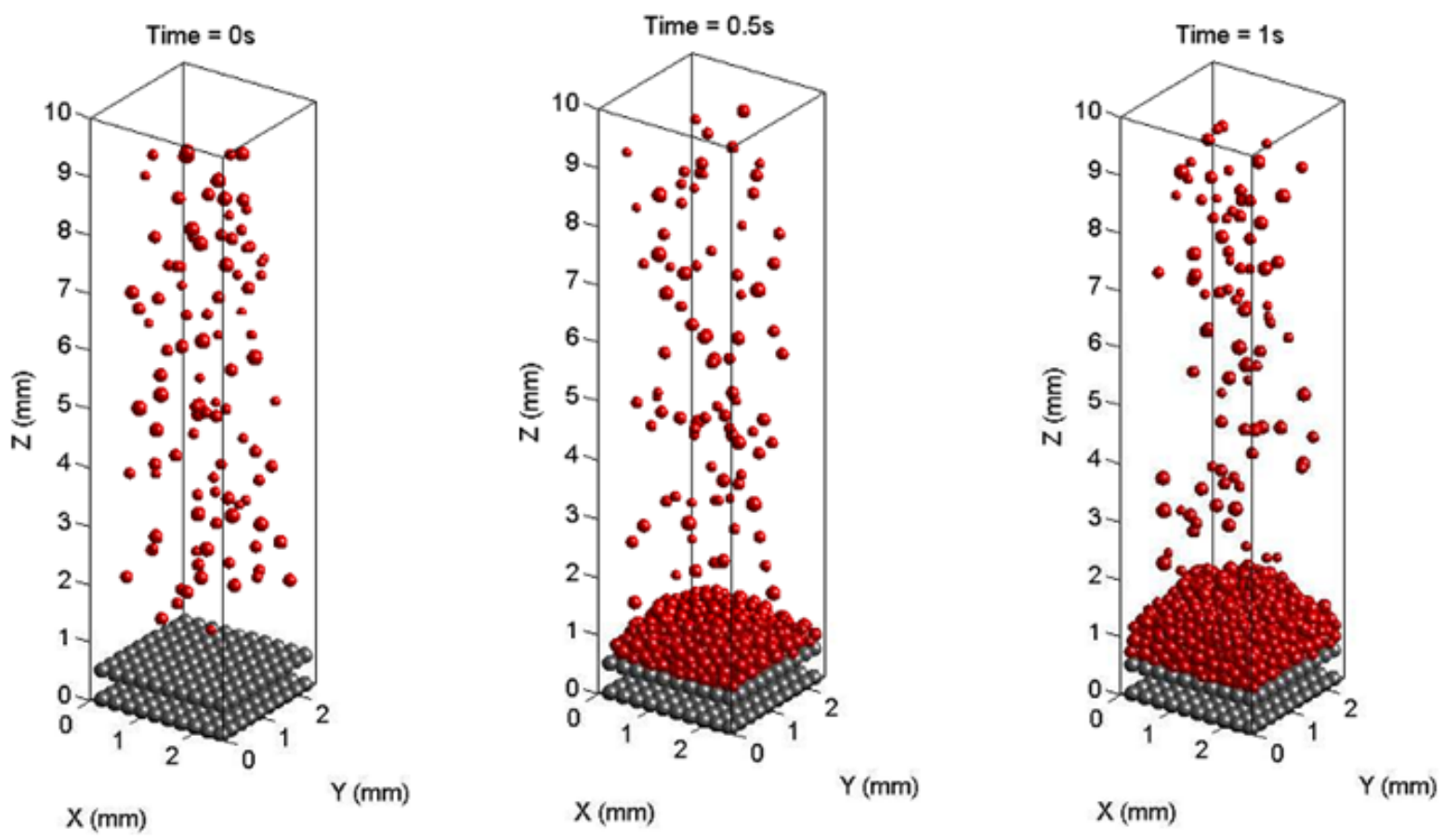

Figure 14. Simulation of the dynamics $(0 \mathrm{~s}, 0.5 \mathrm{~s}$, and $1 \mathrm{~s}$, from left to right) of the constant pressure filtration process with spherical particles $\left(d_{p}=150-250 \mu \mathrm{m}\right)$.

filtering medium up to one second of process simulation, creating an apparently stable bed.

As the filtration process continues and the particles bed is growing, the filtrate flow decreases to keep the pressure drop constant in the filtration cell. Considering that the filtration system simulated in this paper consists of an isotropic and homogeneous porous medium that is percolated by a Newtonian fluid with uniform velocity field, Darcy's equation (Massarani, 2002) was used to describe the dynamic behavior of the filtrate flow (equation 17). The permeability of the cake was obtained by using the Kozeny-Carman equation (equation 18).

$q(t)=-\frac{k_{c}(t)}{\mu_{l}} \frac{\Delta P}{T_{c}(t)}$

$k_{c}(t)=\frac{\phi_{c}(t)^{3} d_{p}^{2}}{180\left(1-\phi_{c}(t)\right)^{2}}$

The simulation results for the temporal profiles of the cake thickness and porosity are shown in Figures 15 and 16, respectively. Figures 17 and 18 exhibit the correspondent profiles for the cake permeability and filtration rate, respectively, obtained from of equations (17) and (18).
Considering the simulation results presented in this section, it is possible to note that as the cake thickness increases, the cake porosity decreases sharply at the beginning of the filtration and reaches a stable value of approximately $40 \%$. As a consequence, the same trend is observed for the cake permeability, which reaches a minimum value of $3.133 \times 10^{-15} \mathrm{~m}^{2}$. As expected, the filtration rate starts dropping around $0.2 \mathrm{~s}$ to keep the pressure drop in the system constant, and reaches a final value of $6.53 \mathrm{~mm} / \mathrm{s}$.

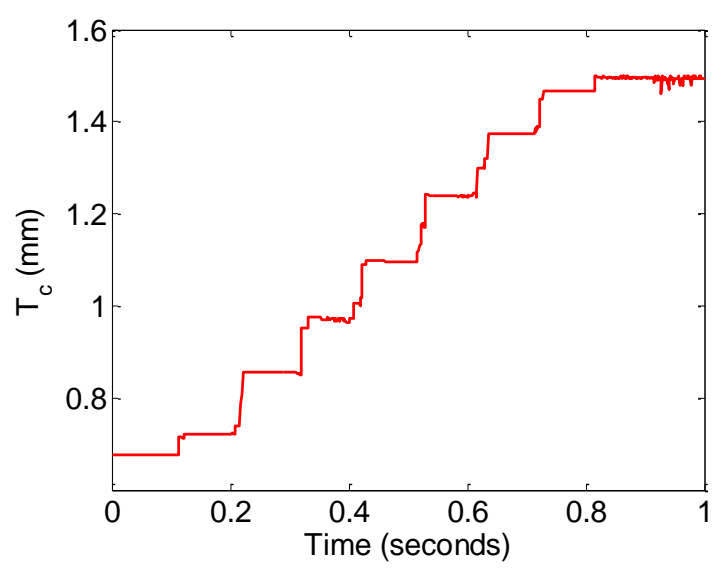

Figure 15. Evolution of the cake thickness with time during the static filtration process. 


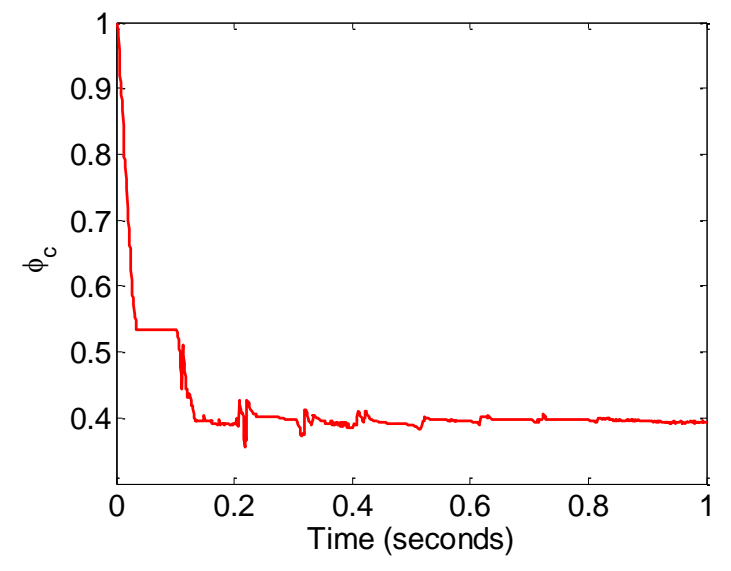

Figure 16. Evolution of the cake porosity with time during the static filtration process.

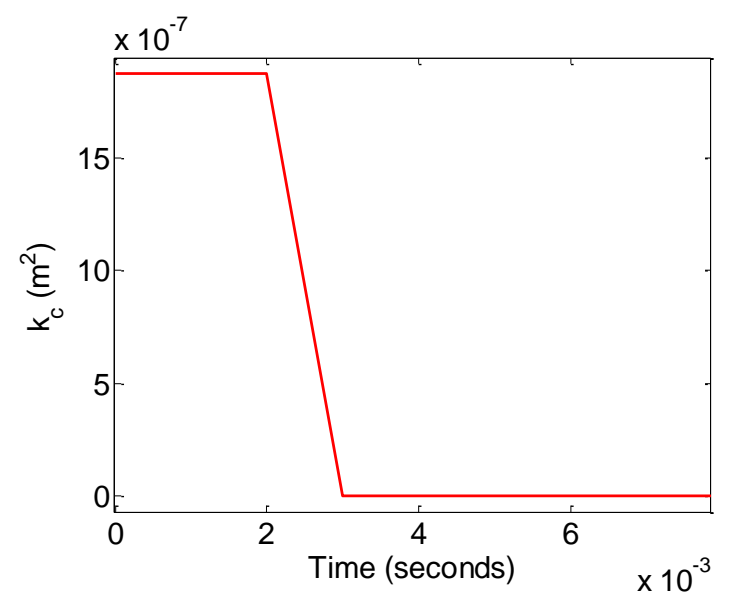

Figure 17. Evolution of the cake permeability with time during the static filtration process.

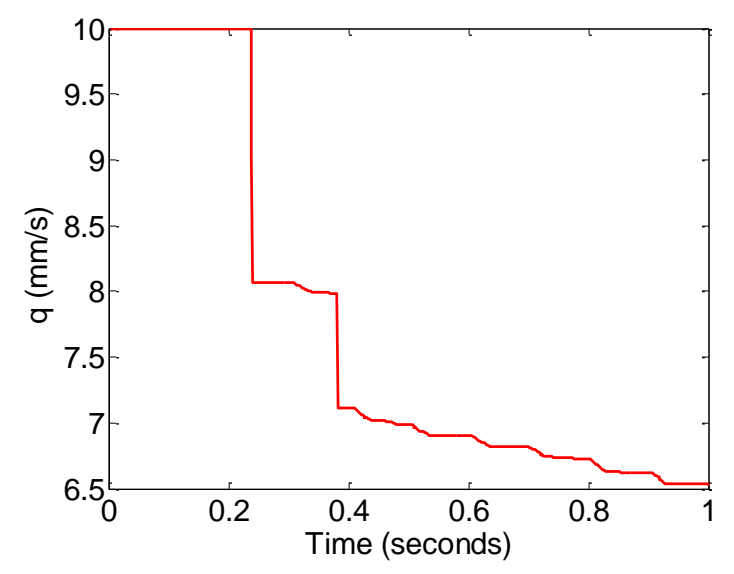

Figure 18. Filtration rate as a function of time during the static filtration process.

This qualitative behavior is corroborated by other results reported in the literature, such as the works by Dong et al. (2003; 2009) and Svarovsky (2000).

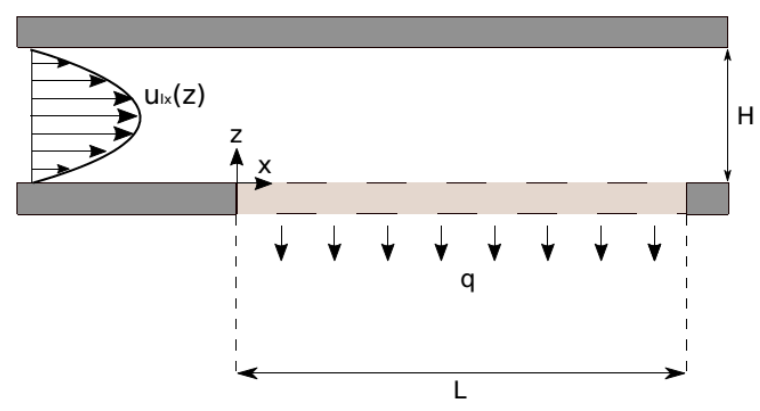

Figure 19. Schematic diagram illustrating the crossflow filtration system in 2D.

\subsection{Simulation of the cross-flow filtration processes}

The last case study simulated in this work was the cross-flow or dynamic filtration process, operating at constant pressure drop and using the same set of physical conditions described in the static filtration case (see Table 3 ). The solid particles were also introduced gradually in the simulated region at a constant rate of 0.00125 particles per time step in an attempt to virtually reproduce the continuous operation mode.

The filter channel was constructed with two parallel plates (rigid boundaries), with the bottom one being permeable to the fluid. It has a height $H$, a length $L$, and a width $W$ (see Figure 19).

The surface of the filtering medium was modeled as a layer of fixed solid particles, with the same mechanical properties of the suspended ones and a diameter of $500 \mu \mathrm{m}$.

According to Hwang and Wang (2001), in most cross-flow filtration systems the cross-flow velocity is far larger than the permeating flow, which allows its variation in the $x$ direction to be reasonably ignored. Thus, in this study the velocity distribution was assumed to be uniform and equal to the filtrate flow rate, $q(t)$, in the permeating direction $(z)$, and parabolic in the cross-flow direction $(x)$, according to equation (19).

$u_{l x}(z)=6\left\langle u_{l x}\right\rangle\left[\left(\frac{z}{H}\right)-\left(\frac{z}{H}\right)^{2}\right]$

where $H$ is the height of the filter channel, $\left\langle u_{f x}\right\rangle$ is the average cross-flow velocity, $u_{f x}$ is the velocity distribution in the cross-flow direction, and $z$ is the vertical position. 

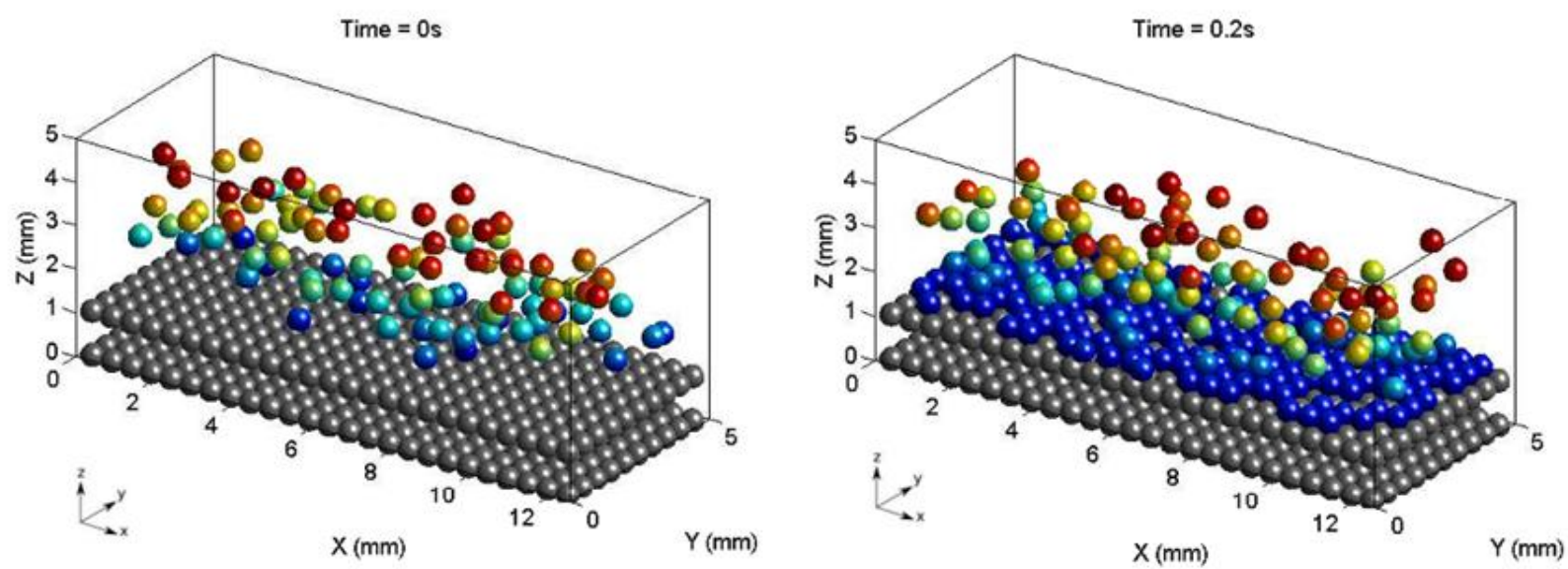

Figure 20. Beginning of the simulation of the cross-flow filtration of 1000 particles.
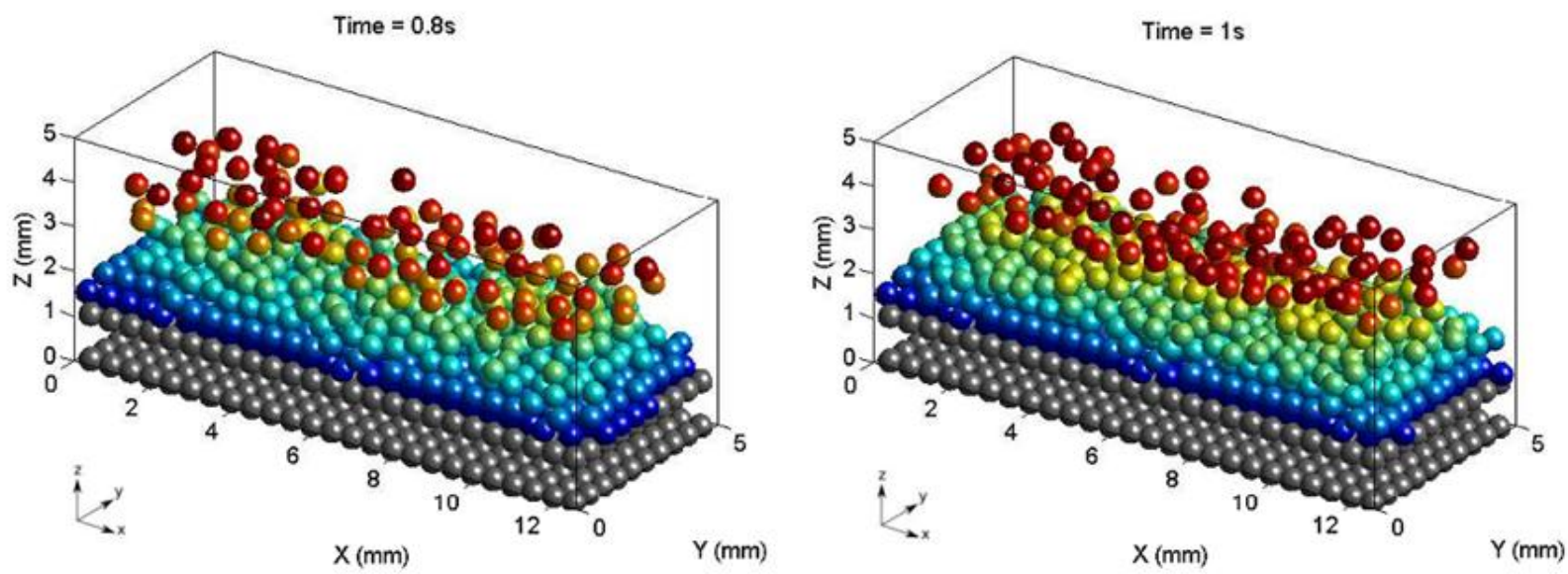

Figure 21. Endo of the simulation of the cross-flow filtration of 1000 particles.

Based on a system comprising 1000 monodispersed spherical particles with $500 \mu \mathrm{m}$ diameter, Figures 20 and 21 present the different time instants $(0 \mathrm{~s}, 0.2 \mathrm{~s}, 0.8 \mathrm{~s}$ and $1 \mathrm{~s})$ of the simulated filtration process operating at a constant pressure drop of $2 \mathrm{kPa}$. The initial filtrate flow rate, $q_{0}$, was considered to be $1 \mathrm{~mm} / \mathrm{s}$ and the average cross-flow velocity, $\left\langle u_{f x}\right\rangle$, was assumed to be 0.1 $\mathrm{mm} / \mathrm{s}$.

According to these diagrams, despite the erosive effect created by the additional component of the drag force in the $x$ direction, a large number of particles (continuously fed in the system) has already settled on the surface of the filtering medium up to one second of process simulation, creating an apparently stable bed.
As in the case of the static filtration process, during the course of the simulation the particles gradually settle on the surface of the porous medium beneath them and the cake starts to grow in thickness. Consequently, the filtrate flow rate, $q(t)$, decreases to keep the pressure drop constant in the filtration cell. Again, Darcy's equation (equation 17) was used to describe the dynamic behavior of the filtrate flow rate through time, and the permeability of the cake was obtained by using the Kozeny-Carman equation (equation 18).

The simulation results for the temporal profiles of the cake thickness and porosity are shown in Figures 22 and 23, respectively. Figures 24 and 25 exhibit the correspondent profiles for the cake permeability and filtration rate, respectively, obtained from of equations (17) and (18). 


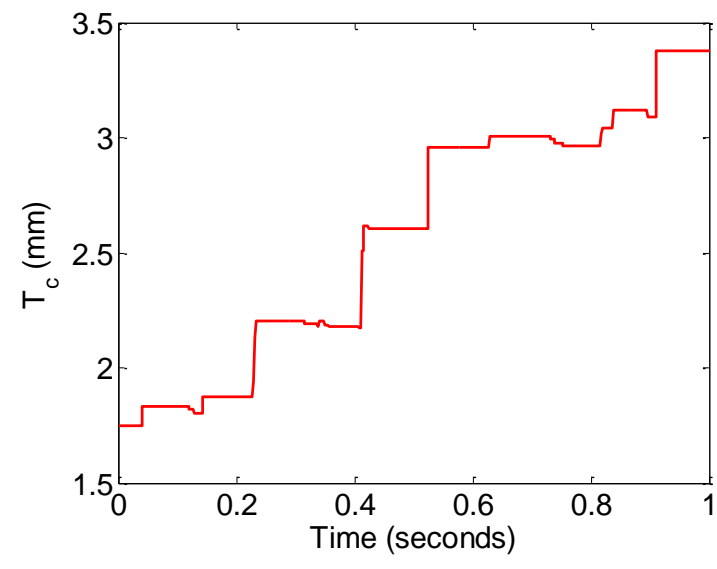

Figure 22. Evolution of the cake porosity with time during the static filtration process.

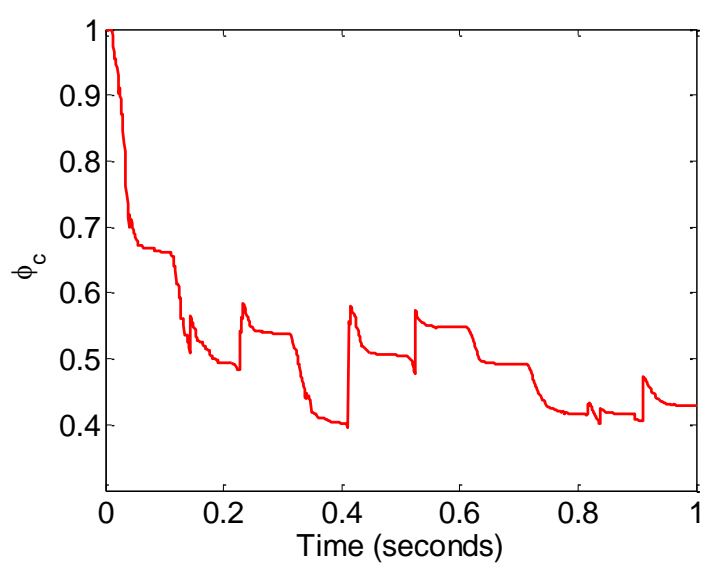

Figure 23. Evolution of the cake porosity with time during the dynamic filtration process.

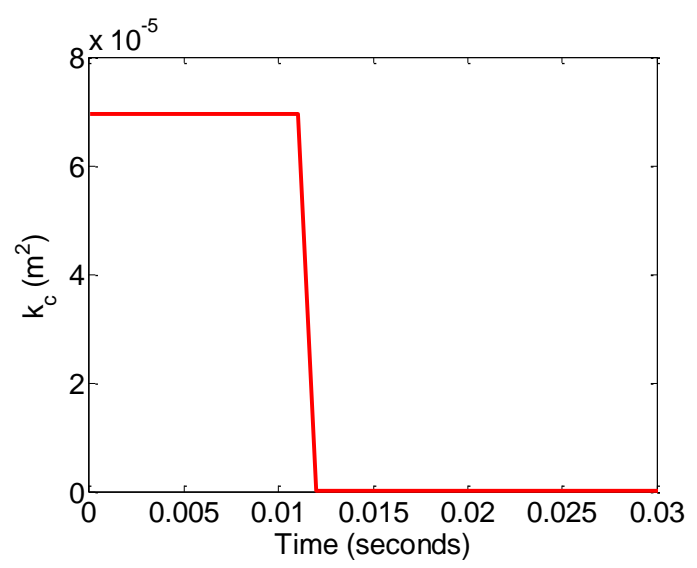

Figure 24. Evolution of the cake permeability during the dynamic filtration process.

Analyzing the simulation results presented above, it is possible to note that as the cake thickness increases, the cake porosity tends to decrease sharply with time, presenting a more oscillatory behavior when compared to static

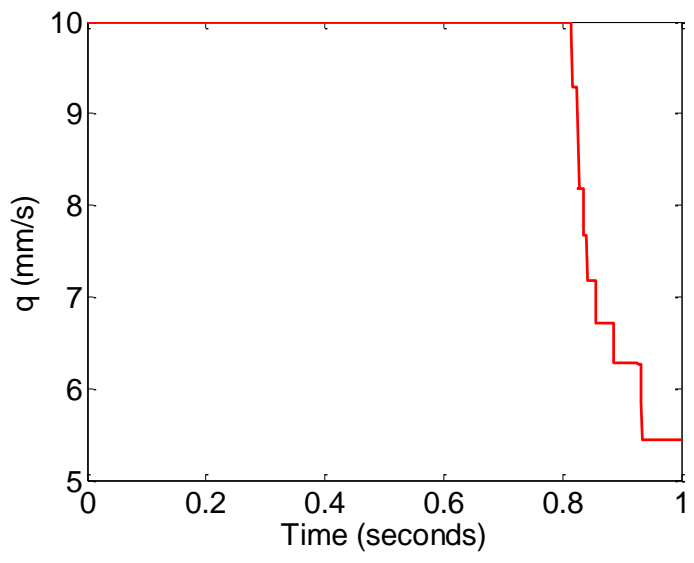

Figure 25. Evolution of the filtrate flow rate during the dynamic filtration process.

filtration case (Figure 16) and reaching a final value of approximately $43 \%$. Again, the cake permeability predicted by the Kozeny-Carman model considerably decreases at the beginning of the simulation, after the first particles start to deposit stably over the porous medium, and, then, it reaches a minimum and a constant value of approximately $1.678 \times 10^{-13} \mathrm{~m}^{2}$.

In this case, only after a cake with reasonable thickness is formed, the filtration rate starts dropping around $0.8 \mathrm{~s}$ to keep the pressure drop in the system constant, reaching a final value of 5.45 $\mathrm{mm} / \mathrm{s}$.

This behavior is qualitatively in good agreement with other results reported in literature, such as the work by Hwang and Wang (2001).

\section{CONCLUSIONS}

The modeling and simulation approach proposed in this work, based on the discrete element method, was successfully employed, since it was possible to describe and analyze the formation and growth of the cake in the sedimentation and filtration processes for a discrete system.

From the microdynamic information, it was also possible to obtain properties such as thickness, porosity, and permeability of the cake over time, which showed a dynamical behavior qualitatively close to the results previously reported in the literature, such as the works by Dong et al. (2006) and Svarovsky (2000). 
In addition, the steady-state results for the cake porosity in sedimentation processes obtained in this work (13 fluids with different densities) were compared to the results reported by Dong et al. (2006) under similar simulation conditions and presented a satisfactory agreement, with deviations less than $10 \%$ for most of the fluids.

\section{ACKNOWLEDGMENTS}

The authors would like to thank CENPES (PETROBRAS Research Center) (4600293210) for the financial support for the project development, and the scientific support from members of PPGEQ/UFRRJ. The support provided by the Formation Evaluation Joint Industry Research Consortium at The University of Texas at Austin is also gratefully acknowledged.

\section{NOMENCLATURE}

A: Hamaker constant $(J)$;

$d_{p}$ : particle diameter $(\mu m)$;

$\boldsymbol{F}_{i j}{ }^{c}$ : contact force acting on particle $i$ by particle $j$ $\left(\right.$ kgms $\left.^{-2}\right)$;

$F_{i j}{ }^{n c}$ : non-contact force acting on particle $i$ by particle $j\left(\mathrm{kgms}^{-2}\right)$;

$F_{i j}{ }^{v}$ : van der Waals force acting on particle $i$ by particle $j\left(\mathrm{kgms}^{-2}\right)$;

$\boldsymbol{F}_{i}^{f}$ : particle-fluid interaction force on particle $i$ $\left(\mathrm{kgms}^{-2}\right)$;

$F_{i j}^{c, n}$ : normal contact force acting on particle $i$ by particle $j\left(\right.$ kgms $\left.^{-2}\right)$;

$F_{i j}^{c, t}$ : tangential contact force acting on particle $i$ by particle $j\left(\mathrm{kgms}^{-2}\right)$;

$\boldsymbol{g}$ : gravitational acceleration $\left(m s^{-2}\right)$;

$h$ : surface gap between two spheres $(\mathrm{nm})$;

$h_{\min }$ : minimum separation distance between two interacting particles $(\mathrm{nm})$;

$k_{c}$ : cake permeability $\left(m^{2}\right)$;

$q$ : filtration rate $\left(m s^{-1}\right)$;

$q_{0}$ : initial filtration rate $\left(m s^{-1}\right)$;

$R_{i}$ : particle radius $(\mu m)$;

$T_{c}$ : cake thickness $(\mathrm{mm})$;

$\boldsymbol{u}_{i}$ : liquid velocity $\left(m s^{-1}\right)$;

$u_{\mid x}$ : liquid velocity component in the $x$ direction $\left(m s^{-1}\right)$

$\left\langle u_{\mid x}\right\rangle$ : average cross-flow velocity $\left(m s^{-1}\right)$;

$Y$ : Young's modulus $\left(\mathrm{Nm}^{-2}\right)$;

\section{Greek Letters}

$\xi_{n}$ : relative normal displacement at contact $(\mathrm{nm})$;

$\xi_{t}$ : relative tangential displacement at contact $(\mathrm{nm})$;

$\mu_{1:}$ liquid viscosity $(c P)$;

$\rho_{l}$ : liquid density $\left(\mathrm{kgm}^{-3}\right)$;

$\rho_{s}$ : solid density $\left(\mathrm{kgm}^{-3}\right)$;

$\phi_{i}$ : local porosity around particle $i$ (dimensionless);

$\phi_{c}$ : cake porosity (dimensionless);

$\omega_{n}$ : component of the relative angular velocity in the contact plane $\left(s^{-1}\right)$;

$\Delta \mathrm{P}$ : applied filtration pressure $(\mathrm{kPa})$.

\section{REFERENCES}

Abreu, C. R. A.; Tavares, F. W.; Castier, M. Influence of particle shape on the packing and on the segregation of spherocylinders via Monte Carlo simulations. Powder Technology, v.134, p. 167180, 2003. https://doi.org/10.1016/500325910(03)00151-7

Allen, M. P.; D. J. Tildesley. Computer Simulation of Liquids. Clarendon Press, 1987.

Coulson, J. M.; Richardson, J. F. Chemical Engineering: Particle Technology and Separation Processes. Butterworth Heinemann, Vol. 2, 1991.

Crowe, C. T.; Schwarzkopf, J. D.; Sommerfeld, M.; Tsuji, Y. Multiphase Flows with Droplets and Particles, $2^{\text {nd }}$ Edition. CRC Press, 2012. https://doi.org/10.1201/b11103

Di Renzo, A.; Di Maio, F. P. Comparison of the contact-force models for the simulation of collisions in DEM-based granular flow codes. Chemical Engineering Science, v.59, p. 525-541, 2004. https://doi.org/10.1016/i.ces.2003.09.037

Dong, K. J.; Zou, R. P.; Yang, R. Y.; Yu, A. B.; Roach, G. Simulation of cake formation and growth in sedimentation and filtration. In: $3^{\text {rd }}$ International Conference on CFD in Minerals and Process Industries, 2003, Melbourne, Proceedings CFDMPI'03, Australia.

Dong, K. J.; Yang, R. Y.; Zou, R. P.; Yu, A. B. Role of Interparticle Forces in the Formation of Random Loose Packing. Physical Review Letters. A. B., v. 96, 145505, 2006.

https://doi.org/10.1103/PhysRevLett.96.145505 
Dong, K. J.; Zou, R. P.; Yang, R. Y.; Yu, A. B.; Roach, G. DEM simulation of cake formation in sedimentation and filtration. Minerals Engineering, v.22, p. 921-930, 2009.

https://doi.org/10.1016/i.mineng.2009.03.018

Hamaker. H. C. The London-van der Waals attraction between spherical particles. Physica, v.4, Issue 10, p. 1058-1072, 1937.

https://doi.org/10.1016/50031-8914(37)80203-7

Hwang, K. J.; Wang, Y. S. Numerical Simulation of Particle Deposition in Cross-Flow Microfiltration of Binary Particles. Tamkang Journal of Science and Engineering, v.4, p. 119-125, 2001.

Israelachvili, J. Intermolecular and Surface Forces. Academic Press, 1991.

Jin, G.; Patzek, T. W. Physics-base Reconstruction of Sedimentary Rocks. In: SPE International Symposium on Oilfield Chemistry, 2003, Proceedings SPE 83587, Long Beach, USA. https://doi.org/10.2118/83587-MS

Langston, P. A.; Tüzün, U.; Heyes, D. M. Discrete Element Simulation of Granular Flow in 2D and 3D Hoppers: Dependence of Discharge Rate and Wall Stress on Particle Interactions. Chemical Engineering Science, v.50, p. 967-987, 1995. https://doi.org/10.1016/0009-2509(94)00467-6

Massarani, G. Fluidodinâmica em Sistemas Particulados. 2a Ed. Rio de Janeiro: E-papers, 2002. (In Portuguese)

Matuttis, H. G.; Chen, J. Understanding the Discrete Element Method: Simulation of NonSpherical Particles for Granular and Multi-Body Systems. John Wiley \& Sons, 2014. https://doi.org/10.1002/9781118567210

Munjiza, A.; Andrews, K. R. F. NBS Contact Detection Algorithm for Bodies of Similar Size. International Journal for Numerical Methods in Engineering, v.43, p. 131-149, 1998.

https://doi.org/10.1002/(SICI)1097-

0207(19980915)43:1<131::AID-NME447>3.0.CO;2-S

Ni, L. A.; Yu, A. B.; Lu, G. Q.; Howe, T. Simulation of the cake formation and growth in cake filtration. Minerals Engineering, v.19, p. 1084-1097, 2006. https://doi.org/10.1016/i.mineng.2006.03.012
Perry, R. H.; Green, D. W.; Maloney, J. O. Perry's Chemical Engineer's Handbook, 7th Edition. Mc Graw-Hill, 1999.

Pöschel, T.; Schwager, T. Computational Granular Dynamics: Models and Algorithms. Springer-Verlag, 2005.

Svarovsky, L. Solid-Liquid Separation. Butterworth Heinemann, 2000.

Xu, B. H.; Yu, A. Numerical simulation of the gassolid flow in a fluidized bed by combining the discrete particle method with computational fluid dynamics. Chemical Engineering Science, v.52, p. 2785-2809, 1997. https://doi.org/10.1016/500092509(97)00081-X

Xu, B. H.; Yu, A.; Chew, S. J.; Zulli, P. Numerical Simulation of the gas-solid flow in a bed with lateral gas blasting. Powder Technology, v.109, p. 13-26, 2000. https://doi.org/10.1016/\$00325910(99)00223-5

Yang, R. Y.; Zou, R. P.; Yu, A. B. Computer simulation of the packing of fine particles. Physical Review E, v.62, p. 3900-3908, 2000.

https://doi.org/10.1103/PhysRevE.62.3900

Zhou, Y. C.; Wright, B. D.; Yang, R. Y.; Yu, A. B. Rolling Friction in the dynamic simulation of sandpile formation. Physica A, v.269, p. 536-553, 1999. https://doi.org/10.1016/S0378-4371(99)00183-1

Zhu, H. P.; Wu, Y. H.; Yu, A. B. Discrete and Continuum Modeling of Granular Flow. China Particuology, v.6, p. 354-363, 2005. https://doi.org/10.1016/S1672-2515(07)60215-2

Zhu, H. P.; Zhou, Z. Y.; Yang, R. Y.; Yu, A. B. Discrete Particle Simulation of Particulate Systems: Theoretical Developments. Chemical Engineering Science, v.62, p. 3378-3396, 2007.

https://doi.org/10.1016/i.ces.2006.12.089

Zhu, H. P.; Zhou, Z. Y.; Yang, R. Y.; Yu, A. B. Discrete Particle Simulation of Particulate Systems: A review of major applications and findings. Chemical Engineering Science, v.63, p. 5728-5770, 2008. https://doi.org/10.1016/j.ces.2008.08.006 